\title{
L'organisation du travail en géotechnique : développement, normalisation et artisanat
}

\section{J.-P. MAGNAN}

Laboratoire central des ponts et chaussées

58 , bd Lefebvie 75732 Paris Cedex 15

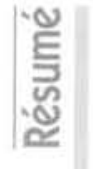

Cet article examine les raisons qui conduisent à consídérer la géotechnique comme un métier autant qu'une collection de disciplines scientifiques. Ce métier ne peut être découpé en tâches élémentaires et s'apparente par beaucoup d'aspects à l'artisanat. Cela conduit à des conditions spéciales pour l'exercice de professions qui peuvent être séparées dans d'autres domaines : enseignants et chercheurs doivent maîtriser l'exercice du métier géotechnique. Les normes tiennent comptent aussi des spécificités de la géotechnique et laissent une large place à la responsabilité individuelle des géotechniciens.
NDLE: Les discussions sur cet article sont acceptées jusqu'au 31 décembre 2002
This paper discusses the reasons why geotechnical engineering should be considered as a craft as much as a collection of scientific disciplines. This type of activity cannot be split into elementary tasks and it has many ommon features with craftsmanship. This results in a profession, which cannot be divided, as it is possible in other fields: teachers and research workers must have a thorough knowledge of geotechnical engineering and leave much room for the personal responsibility of geotechnical engineers. 
"Because nature is infinitely variable, the geological aspects of our profession assure us that there will never be two jobs exactly alike. Hence we need never fear that our profession will become routine or dull. If it should, we would not be practising properly, " (Presidential addiress, 8th ICSMFE, Moscow, 1973, 4,1, 156-159).

\section{Préambule}

Cet article suggère une réponse globale à diverses interrogations sur l'avenir de la profession géotechnique : dans ses relations avec la société, dans ses relations avec le monde économique, dans ses relations avec les autres professions du génie civil, de l'environnement, de l'industrie des mines et du pétrole, dans les relations entre les métiers d'enseignants, chercheurs, concepteurs, experts... qui sont parfois distingués en son sein. Il répond aussi à l'inquiétude de nos collègues des bureaux d'études comme des entreprises, du fait que l'organisation économique entraine un découpage d'activités dont l'interpénétration a toujours été un facteur essentiel de succès et de progrès. Une émission consacrée à John Ford, à Frederick W. Taylor et à l'organisation scientifique du travail a suggéré une clé de lecture : l'organisation du travail avec un modèle qui, tout compte fait, parait le plus proche de nos besoins : l'artisanat. Cela a conduit ensuite à analyser l'adéquation de ce modèle à la réalité de la géotechnique et comment il interagit avec le développement et la codification en cours des activités d'essais, de calcul et d'exécution des travaux. Le lecteur ne trouvera pas dans ce texte de commentaires détaillés du champ de la géotechnique, car il traite plus du métier de géotechnicien que de la qéotechnique. Si les exemples présentés concernent plutôt les projets d'ouvrages, dans la problématique générale de l'Eurocode 7, la situation est identique pour la géotechnique appliquée aux risques naturels, où l'expérience joue aussi un rôle décisif dans la compétence des géotechniciens et où le retour d'expérience est d'une importance majeure. Elle l'est aussi, de façon générale, pour la géologie de l'ingénieur.

\section{1}

\section{Quelques définitions}

Même si réfléchir sur l'organisation du travail n'est pas une activité scientifique au sens usuel du terme, notre raisonnement suivra la mème démarche générale : définir l'objet de l'étude, puis l'analyser de différents points de vue. Deux définitions peuvent être utiles à la compréhension de notre discours et nous poursuivrons par l'énumération de quelques tendances actuelles qui influencent le fonctionnement de la géotechnique, en France et dans les pays qui sont organisés de façon comparable.

\section{Géotechnique}

La première définition est celle du mot géotechnique. Pour nous, c'est un champ d'activité profession- nelle, correspondant aux mots anglais geotechnical engineering et que l'on aurait pu aussi appeler " génie géotechnique m. La géotechnique recouvre aussi bien les études expérimentales que les études théoriques, la conception et l'exécution des ouvrages, les interactions des sols et des structures, la reconnaissance des sites, les études de matériaux naturels, les risques naturels, les problèmes de pollution des sols... A ce titre, elle utilise les outils de la mécanique des sols, de la mécanique des roches et de la géologie de l'ingénieur. Ses voisins sont la science du sol, proche de l'agriculture et intéressée par le premier mètre de la croûte terrestre, et les génies pétrolier et minier, qui ont des objets plus profonds.

La géotechnique porte un double regard, à la fois naturaliste et mécanique, sur ses objets : elle utilise et développe des modèles mécaniques, mais pour des matériaux naturels qu'elle ne peut complètement caractériser et qui sont souvent perturbés par l'exécution des travaux. La géologie contribue à la compréhension des sites des études et des propriétés physico-chimiques et mécaniques des sols et des roches. L'observation et l'analyse du comportement des sites et des ouvrages ont une importance décisive pour la validation des méthodes de conception et de construction, qui évoluent rapidement avec le progrès technique.

C'est à dessein que nous n'utilisons pas les mots de " géomécanique », parce que l'individualisation des analyses issues de la mécanique théorique ne nous parait pas souhaitable hors du contexte de la géologie et du réel, ni de " géomatériaux», dont l'invention pour regrouper des matériaux naturels avec des matériaux issus de processus industriels ne nous parait pas fondée, ni de "poromécanique » ou "mécanique des milieux poreux n, qui désignent une branche formalisée de la mécanique des milieux continus déformables dont le développement est intégré depuis longtemps à la mécanique des sols.

\section{2}

\section{Artisanat et taylorisme}

Nous avons consulté quelques dictionnaires pour comparer des définitions de ces termes. On trouve à $\alpha$ Taylor , dans le Petit Robert 2 : $* I l$ fut, sinon l'initiateur du travail à la chaîne, du moins le promoteur de l'organisation scientifique du travail industriel (taylorisme) ; celle-ci suppose (citation de G. Friedman) l'utilisation maximale de l'outillage, la spécialisation stricte et la suppression des gestes inutiles, ainsi qu'un système de primes pour inciter l'ouvrier au rendement. Cette méthode favorisa l'augmentation de la production, mais conduisit sous le couvert de la simplification et de l'économie des gestes à dépouiller les tâches de connaissances professionnelles, de qualification, d'initiative.

L'artisan est défini par le dictionnaire usuel QuilletFlammarion (1963) comme «celui qui exerce un métier manuel, un art mécanique pour son propre compte» ou «ouvrier qualifié qui travaille chez lui » et par le dictionnaire Hachette (Bibliorom Larousse) 2000 comme "personne qui exerce pour son propre compte un art mécanique ou un métier manuel). L'artisanat est (cla profession d'artisan ou l'ensemble des artisans » (Quillet-Flammarion) ou « la profession d'artisan, l'ensemble des artisans, la technique de l'artisan ou la production artisanale $»$ (Hachette). L'art à son tour est défini comme "application d'un ensemble de connais- 
sances théoriques et techniques à une réalisation pratique $n$ (Quillet-Flammarion) ou "ensemble de connaissances, de techniques nécessaires pour maîtriser une pratique donnéen (Hachette).

Mais on trouve dans un dictionnaire plus ancien (Nouveau petit Larousse illustré, 1929) une définition de l'artisan qui correspond mieux au sens que nous donnons à artisanat dans le titre du présent document : " homme de métier , avec cette idée que la géotechnique doit être d'abord pensée comme un métier. Le métier est défini dans ce dictionnaire comme «toute profession manuelle ou mécanique» ou "une profession quelconque $n$.

L'opposition de l'artisanat à l'organisation scientifique du travail n'est pas fortuite. L'artisanat repose sur la compétence professionnelle de l'artisan et la maitrise complète de son art. Le perfectionnement des outils dans la perspective de l'artisanat se traduit par plus de souplesse et de plus grandes possibilités d'adaptation à des situations différentes. A l'opposé, le développement des machines dans la perspective de l'organisation du travail s'intègre dans une conception spécificatrice des produits du travail, permettant de produire en grand nombre des objets tous identiques, avec des opérateurs successifs ne connaissant pas l'ensemble du processus : les machines se perfectionnent mais pour des tâches qui restent prédéfinies. Les deux modes de production peuvent coexister, suivant la nature des objets à produire, mais ils restent marqués par des logiques différentes.

L'affinité de l'artisanat et de la géotechnique trouve son origine dans la nature même des études géotechniques, qui ne portent jamais deux fois sur le même objet, et dans le rôle essentiel de l'expérience comme élément fondateur de la compétence du géotechnicien. Elle illustre le fait que l'étude géotechnique n'est pas un produit industriel, même si certaines parties du travail peuvent être organisées de façon industrielle.

\section{2}

\section{Tendances actuelles}

Ce panorama introductif ne serait pas complet sans un inventaire des éléments qui conditionnent la situation et l'évolution actuelle de la géotechnique. Cet inventaire est partiel, parce qu'il s'applique d'abord au contexte français, mais beaucoup de points sont communs à la géotechnique internationale.

La première constatation est que les décideurs ont perdu la conscience des contraintes de la nature. Le progrès technologique donne l'impression que tout problème géotechnique peut être traité rapidement, à condition de mettre en place le financement nécessaire. La géotechnique ne reçoit plus d'attention particulière dans les projets.

La deuxième constatation est que la gestion intégrée des projets (maîtrise d'ouvrage, maîtrise d'œuvre, bureau d'études, surveillance du chantier), qui a fait la force de l'ingénierie (publique) française au cours des cinquante dernières années et permis un développement remarquable des entreprises, confrontées à des clients-partenaires compétents et forts, a été fortement contestée pour des motifs non techniques, au profit d'une contractualisation détaillée, qui nécessite de découper le processus de conception et de réalisation des travaux en une série de marchés successifs avec appel méthodique à la concurrence : ainsi, il n'est pas rare que les géotechniciens de la phase des travaux n'aient jamais été mêlés à la conception de l'ouvrage. Toutefois, la pratique des marchés globaux, incluant la conception et la réalisation du projet, offre de nouvelles perspectives, s'il est possible d'y inclure des opérations de gestion du retour d'expérience pour l'ensemble de la communauté géotechnique.

La troisième constatation est que nous sommes entrés dans une période de suspicion généralisée où lon perfectionne les règles juridiques pour mieux surveiller les relations entre les acteurs économiques et ce qui est ressenti dans les milieux économiques comme les conditions normales de concurrence. Ces règles juridiques ont pour effet de limiter les relations techniques permanentes entre acteurs économiques, ce qui n'est pas favorable au maintien et au développement de l'expérience technique, dont nous avons déjà suggéré l'importance en géotechnique.

Dans le contexte français, on observe ainsi une évolution de l'organisation vers la spécialisation de " boites fonctionnelles , chargées de parties de projets et communiquant par des contrats de plus en plus normalisés.

Par ailleurs, l'affirmation du métier de chercheur, par opposition à celui d'ingénieur, c'est-à-dire de spécialiste qui n'est pas partie du processus de production, a conduit à la montée en puissance d'un cadre de recherche où, par principe, le jugement des pairs devient la référence de base et peut remplacer totalement le jugement autrement plus rigoureux de la pratique et du réel. Pour la géotechnique, cette situation est préoccupante.

Enfin, on observe une contestation parallèle, bien que d'origine différente, des structures publiques qui assuraient, pour le bien de tous, une liaison volontariste entre les différents métiers, outils et compétences du monde de la construction.

3

\section{Deux situations différentes: géotechnique et structures}

Pour faire sentir la particularité de la géotechnique parmi les activités des ingénieurs, il nous a paru utíle de la comparer à un autre champ professionnel du monde de la construction, qui est la conception et le calcul des structures. Cette comparaison s'appuie sur des réflexions menées il y a quelques années, lors de la discussion des règles générales du système des Eurocodes structuraux (normes européennes de justification des ouvrages dans le domaine du bâtiment et du génie civil). Il nous avait en effet fallu expliquer à nos collègues issus du monde des structures pourquoi il fallait donner à la géotechnique des ouvertures particulières par rapport au corps de règles qu'ils s'étaient assez unanimement imposées.

Les difficultés rencontrées lors de la préparation des règles communes pour le calcul des structures et le calcul géotechnique en Europe (Eurocodes structuraux) sont illustrées par les schémas des figures 1 et 2 . qui décrivent les raisons de la "différence géotechnique $)$.

Le calcul des structures s'appuie sur un ensemble de règles, enseignées en France sous le nom de «résis- 
tance des matériaux n et complétées par des règles de construction issues de l'expérience. Les propriétés des matériaux sont spécifiées, les charges sont définies de façon séparée et le tout est considéré comme raisonnablement étalonné par rapport à la réalité.

Pour la conception et l'exécution des structures, on comprend donc que l'on puisse isoler des blocs dans le schéma de la figure 1 et les laisser se développer de façon séparée. Cela provient notamment de l'existence depuis les années 60 d'un code modèle européen pour le béton, qui a unifié le formalisme des calculs et a servi de base à l'élaboration de codes nationaux appuyés sur:

- un ensemble unifié de règles de calcul et de dimensionnement

- une description semi-probabiliste des charges appliquées aux structures :

- un mode de caractérisation des matériaux de construction qui s'appuie sur une probabilité contrôlée que leurs propriétés physiques et mécaniques soient moins favorables (plus faibles ou plus fortes) que la valeur spécifiée :

- des règles ou spécifications supplémentaires issues de la pratique de la construction, et

- un ensemble d'essaís d'étalonnage en vraie grandeur sur des structures entières ou des éléments de structures.

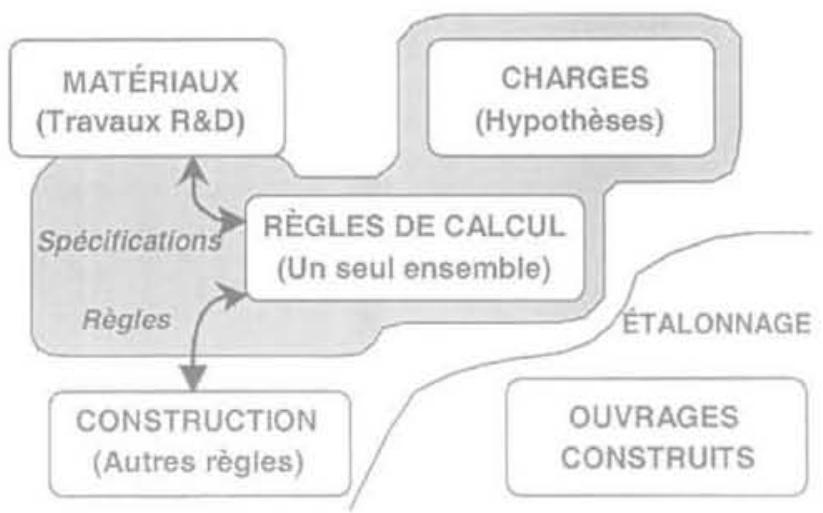

FiG.1 Schéma des méthodes de dimensionnement des structures.

Schematic framework for structural design methods.

Si nous examinons maintenant la pratique de la géotechnique (Fig. 2), il apparaît que les principales différences par rapport au calcul des structures concernent les règles de calcul (dimensionnement) et la description (ou prescription) des matériaux :

- les géotechniciens ont développé beaucoup d'approches différentes pour le dimensionnement des différents types d'ouvrages de la géotechnique, sur la base d'essais de laboratoire et d'essais en place. Certaines de ces méthodes de dimensionnement sont utilisées dans le monde entier, d'autres ne sont connues que localement, mais elles ne peuvent être éliminées pour cette seule raison, surtout quand elles ont été incorporées dans des normes ou codes nationaux. D'autre part, chaque méthode s'appuie sur une expérience locale ou internationale qui compte beaucoup dans sa justification : I'application pratique ne peut être isolée de la définition des règles de calcul

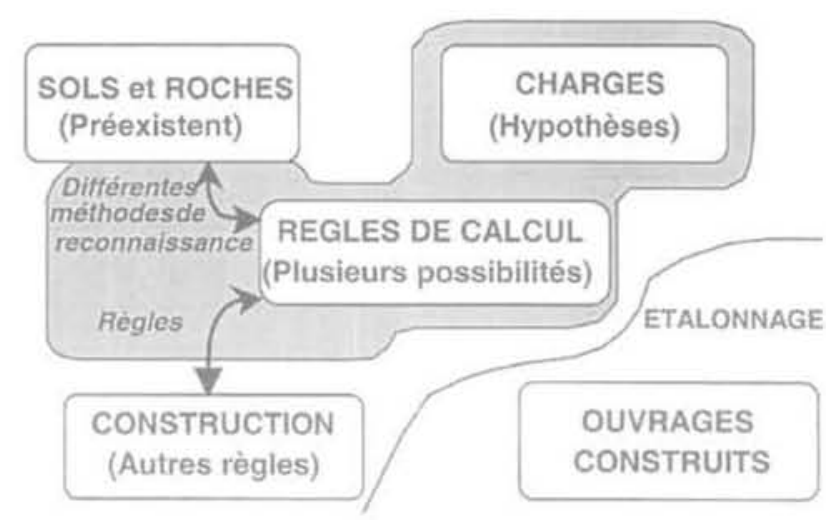

FG ? Schéma des méthodes de dimensionnement des ouvrages géotechniques.

Schematic framework for geotechnical design methods.

- à la différence de l'acier ou du béton, les sols et les roches sont déjà présents sur les sites de construction et ils ne peuvent être étudiés que partiellement. Chaque site est unique. Personne ne peut certifier que la stratification et les propriétés géotechniques du terrain sur un site sont identiques à celles du site voisin.

Ceci a au moins trois conséquences :

- la première est que les codes de géotechnique donnent d'habitude des règles de reconnaissance des sites plutôt que des prescriptions sur les valeurs des paramètres de calcul. Lorsqu'un ingénieur en structures décide de choisir un béton de type B45, il n'a pas à tester le béton, ni même à se préoccuper de la façon dont il va être fabriqué, ni où : il sait précisément que le béton qui sera utilisé pour la construction aura des propriétés mécaniques bien définies, avec une probabilité limitée d'avoir des valeurs inacceptables. Au contraire, lorsqu'un ingénieur géotechnicien doit décider des valeurs de calcul des propriétés d'un sol ou d'une roche, il doit utiliser un très petit nombre de résultats d'essais, qui ne sont pas toujours fiables, et toute autre information qu'il peut trouver sur le site, comme des données géologiques ou des expériences locales : en général, il ne sait pas de combien les valeurs de calcul choisies s'écartent des valeurs optimales, mais il sait seulement que sa façon usuelle de procéder est assez sécuritaire

- la deuxième conséquence est que l'étalonnage des méthodes de dimensionnement est plus compliqué que pour les structures ou éléments de structures en béton ou en acier : comme les sites de construction ne sont pas identiques et, de plus, ne peuvent être parfaitement connus, la fiabilité d'une procédure de dimensionnement (qui inclut la reconnaissance du sol, la méthode de calcul et l'interprétation des résultats du calcul) ne peut être estimée sans tenir compte des incertitudes sur les propriétés des sols ni de la capacité de l'ingénieur à choisir des valeurs adéquates des paramètres de calcul. Pour cette raison, un grand nombre d'expérimentations est nécessaire pour l'étalonnage de chaque méthode de dimensionnement (méthode de reconnaissance $x$ méthode de calcul) ;

- la troisième conséquence est que la conception des ouvrages ne peut généralement être garantie indépendamment de la réalité des terrains que l'on découvre lors du chantier. La méthode dite «observationnelle m, dont l'utilisation est recommandée par tous les maitres de la géotechnique pour les ouvrages com- 
plexes, traduit cette interaction forte entre la conception, l'exécution et la réalité des terrains lors des travaux, qui implique le concepteur géotechnicien, le maître d'œuvre, le maître d'ouvrage et l'entreprise de travaux.

Ces observations justifient la revendication des géotechniciens de ne pas ètre pris dans un système de boites indépendantes ( «règles de calcul », (conception », ((exécution ») gérées par des appels d'offres indépendants. La sécurité et loptimisation technique et économique des ouvrages ne peuvent être obtenues si l'on interdit les interactions entre la reconnaissance des sites, la conception des ouvrages et les observations faites lors des travaux. Il devrait y avoir, pour chaque ouvrage, un contrat d'étude et d'assistance géotechnique, conclu par la maîtrise d'ouvrage et s'imposant à tous les autres intervenants du projet et des travaux, éventuellement complété par des prestations partielles, contrôlées dans le cadre du contrat général.

\section{4}

\section{La géotechnique est un artisanat}

Il n'existe pas de textes en français sur la nature et les missions de la profession géotechnique. Rien de comparable avec, par exemple, l'cuvre de Ralf B. Peck. qui a suscité l'enthousiasme de nombreux jeunes ingénieurs pour ce métier (Dunnicliff et Deere, 1984). Il faut donc rechercher dans notre expérience collective les fondements d'une conception de notre métier de géotechnicien.

Nous avons thabitude en France, depuis plus de cinquante années, d'avoir des bureaux d'études géotechniques intégrant, sous des cadres juridiques divers, des équipes de sondage et des laboratoires d'essais. Ces structures publiques (EdF, Laboratoires des ponts et chaussées) ou privées (CEBTP, Sociétés de Louis Ménard, Fondasol, Simecsol, Sols-Essais...) ont participé activement, par la pratique et la recherche-développement, à la montée en puissance (et en compéterice) de la géotechnique française et à sa reconnaissance internationale. La caractéristique essentielle de ces organismes a été leur capacité à couvrir l'ensemble du champ de la recherche, du développement de théories et de matériels à l'expérimentation, et de l'application dans les projets : un champ d'activité complet pour le géotechnicien et une activité d'ingénieur au sens noble du terme, dans tous ses aspects de création et d'action.

Nous avons eu aussi, pendant la même période, un autre groupe d'activités de recherches théoriques, d'origine universitaire, ayant ses fondements dans la tradition française d'excellence en mathématique et en mécanique, mais sans relations avec la pratique et dont l'influence sur cette pratique a été faible, autant que l'on puisse l'évaluer.

Actuellement, la prééminence des structures économiques sur l'organisation technique de la société favorise la création d'unités économiques comparables aux "boites » dont nous avons déjà parlé : la boîte de la mầtrise d'ouvrage, la boîte des bureaux d'études, la boite des entreprises de sondages, la boite des entreprises de travaux, la boîte des contróleurs techniques, la boîte des enseignants, la boite des chercheurs..., chacune avec son champ de compétence, ses règles et ses personnels.
Cette évolution est contraire aux besoins de la géotechnique, dont les progrès (et même la survie) ne peuvent venir que d'une connaissance complète du champ géotechnique, et du perfectionnement de ses acteurs par la pratique et l'accumulation d'expérience. C'est en ce sens que la géotechnique se compare le mieux avec l'artisanat : chaque géotechnicien est un artisan, qui dispose d'outils plus ou moins complexes, mais dont il décide lui-même de l'usage pour élaborer un projet dont il est responsable, sur un site particulier et pour un ouvrage souvent unique. Les règles dont se sont dotés les géotechniciens au cours des années vont d'ailleurs dans ce sens.

\section{5}

\section{Géotechnique et normalisation}

A part quelques exceptions, comme le Fascicule 62Titre $V$ applicable au calcul des fondations dans les marchés publics en France (1993), les textes régissant la pratique de la géotechnique sont plus des recueils d'indications sur les moyens que des textes édictant des règles précises. D'ailleurs, l'essentiel des règles de la géotechnique est contenu dans des recommandations ou des "codes de bonne pratique».

Le texte en préparation de l'Eurocode 7 (future norme européenne EN 1997-1, prévue pour 2003) respecte cette orientation générale et exprime dès son début des préoccupations de compétence du personnel qui sont la traduction en langage de normalisation de l'affirmation que la géotechnique n'est pas l'application automatique de règles et de recettes, mais nécessite de l'expérience, Il n'a pas été possible d'écrire que la géotechnique devait être pratiquée par des géotechniciens expérimentés, mais c'est le sens de ces phrases. On lit dans la section 1 "Généralités », paragraphe 1.4 "Hypothèses » que :

1) Les dispositions de cette norme sont fondées sur les hypothèses suivantes. Les utilisateurs de la norme feront leur possible pour assurer que :

- les données nécessaires au calcul ont été collectées, enregistrées et interprétées par des personnels possédant une qualification adéquate;

- les ouvrages sont conçus et calculés par des personnels possédant une qualification et une expérience adéquates :

- des relations et communications adéquates existent entre les personnels impliqués dans la collecte des données, la conception, le calcul et la construction :

- une surveillance et un contrôle de qualité adéquats sont assurés dans les usines de préfabrication, les installations de chantier et sur le terrain ;

- l'exécution est effectuée conformément aux normes et spécifications applicables, par des personnels ayant les capacités et l'expérience appropriées ;

- les matériaux de construction et les produits sont utilisès selon les spécifications de l'Eurocode 7-1 ou des textes applicables aux produits ou matériaux concernés ; - l'ouvrage sera entretenu correctement pour durer pendant toute sa durée de service prévue :

- l'ouvrage sera utilisé comme prévu dans le projet.

2) Ces hypothèses doivent être prises en compte à la fois par le concepteur et par le client (maitre d'ouvrage). Pour lever tout doute, la vérification de ces hypothèses devrait ètre notée par écrit, par exemple dans le rapport de calcul géotechnique... 
Plus généralement, la normalisation de la géotechnique, considérée comme un métier, n'est pas plus facile à faire que celle de la médecine, autre profession où le spécialiste doit formuler un diagnostic et proposer des actions sur la base d'indices externes, sans pouvoir disséquer l'objet de son étude.

Si l'on considère que la normalisation a pour mission de définir les caractéristiques d'objets ou d'opérations que l'on pourra ensuite échanger et vendre, la longue liste des normes géotechniques françaises ou étrangères témoigne de la diversité des essais, des ouvrages et des techniques de réalisation que le géotechnicien doit connaitre et peut utiliser. Mais l'expérience que l'on peut tirer des expertises, études de projets ou observations faites sur des ouvrages montre que les normes restent un outil qui ne garantit pas que l'ouvrage construit sera adapté à son usage. Le processus de conception géotechnique suit en effet un cheminement en trois étapes :

- la première étape est la reconnaissance du site. Elle comporte la visite du terrain, des levés géologiques, des sondages, des essais en place et en laboratoire, des reconnaissances géophysiques et produit un modèle géotechnique du site qui sert de base pour la conception de l'ouvrage :

- la deuxième étape est la conception et la justification de l'ouvracie. Elle utilise l'expérience (la connaissance des modèles d'ouvrages courants ou exceptionnels) et la créativité de l'ingénieur, plus des outils et règles de justification :

- la troisième étape est celle du suivi des travaux, qui permet de valider le projet ou de le modifier pour tenir compte de la réalité du site. Les outils du géotechnicien sont alors l'observation visuelle, les mesures de déplacements et de pressions dans le terrain et dans les ouvrages.

On sait normaliser les procédures d'essai, les règles de calcul, l'exécution des travaux, même si cela n'est pas encore fait complètement. Mais on ne sait pas définir a priori le processus intellectuel qui permet de construire le modèle géotechnique du site et de louvrage. Le modèle géotechnique dépend des informations qu'il a été possible de rassembler sur le site et de la compétence (de l'expérience) du géotechnicien. La recherche d'informations sur le site a souvent un coût élevé, car les matériels de sondage et d'essai sont des machines complexes et chères et ces prestations prennent aussi du temps et elle doit se faire au stade des études. L'organisation du financement des opérations de construction est mal adaptée aux besoins de la géotechnique. Au stade des études; les budgets sont peu importants et il est rare qu'ils puissent couvrir toutes les investigations nécessaires (le coût de la géotechnique dans les projets de construction est rarement supérieur à $1 \%$ du coût total de l'ouvrage, dont $80 \%$ pour les essais et sondages. Mais si l'on estime à $10 \%$ du total du projet le coût des études, la géotechnique en représente près de $10 \%$ ). On se trouve donc souvent dans la situation où les données géotechniques sont insuffisantes et le modèle géotechnique peu fiable... sans compter les cas où les sondages et essais ont été ratés ou bâclés, ou ceux oủ le maître d'ouvrage ne donne pas le temps nécessaire à l'exécution des reconnaissances géotechniques. Il est alors indispensable de profiter des autres phases du projet pour vérifier le modèle géotechnique et éventuellement rectifier le projet. Tout ceci montre pourquoi la géotechnique devrait avoir une place à part dans la gestion des pro- jets. Mais cela montre aussi que le géotechnicien doit souvent utiliser son expérience plus que des résultats d'essais.

Comme on ne peut normaliser l'expérience, par essence individuelle et nourrie des travaux de chacun et des exemples relatés dans des livres ou des revues, la normalisation de la géotechnique ne peut être que partielle. Les longs débats entre géotechnique et structures pour la préparation des Eurocodes étaient en fait des débats destinés à préserver la part de l'expérience dans les études géotechniques. Pour prendre un exemple, la question a été débattue de savoir si l'on doit multiplier par 1,35 les efforts transmis par le sol à une structure alors que le géotechnicien pense déjà avoir mis la sécurité nécessaire dans le calcul de ces efforts ou s'il est raisonnable de multiplier par ce mème coefficient 1,35 la masse volumique du sol (dont les écarts types sont très faibles, quelques pour-cent au plus). Le coeur de ce problème, du point de vue de la géotechnique, est que les hypothèses des calculs qéotechniques ne sont pas seulement des résultats d'essais fiables et en nombre suffisant pour faire des analyses statistiques : dans beaucoup de cas, les hypothèses des calculs sont issues de l'expérience et, si l'on veut modifier les coefficients des équations prouvant la sécurité d'un ouvrage, il faut pouvoir expliquer à chaque géotechnicien comment il doit modifier l'usage qu'il fait de son expérience personnelle. Ce n'est bien sûr pas facile.

Quelle est la place de la normalisation, dans un tel contexte? Elle existe : il faut disposer de modes opératoires communs pour les sondages et les essais. Il est bon de définir les procédures de justification des ouvrages, mais en gardant à l'esprit que la sécurité provient à la fois des facteurs de sécurité et du modèle géotechnique du site. Il est bon aussi de définir les grandes règles des procédures d'exécution des travaux. Nous avons enfin en France une norme originale qui définit les missions géotechniques (NF P 94-500). Mais nous n'avons pas de procédure de qualification des compétences des géotechniciens, à l'instar de ce qui existe dans de nombreux pays, malgré l'importance du facteur humain dans la pratique de la géotechnique. Et nous n'avons pas non plus de procédures de mesure et donc de comparaison de la qualité des prestations fournies. Si nous ajoutons la revendication d'une géotechnique associée à la maitrise d'ouvrage pour l'ensemble du projet, il reste donc un vaste espace à organiser pour que la place de la géotechnique dans la société soit reconnue à sa juste importance et préservée.

\section{6}

\section{Géotechnique et recherche}

Le plaidoyer des paragraphes précédents pour une géotechnique intégrée, oủ le métier de géotechnicien est l'épine dorsale d'un système reliant la reconnaissance des sites, la conception des ouvrages et le suivi des travaux, s'applique aussi à la recherche. Les instituts de recherche en géotechnique doivent être aussi des organismes reconnus pour leur compétence générale en géotechnique, comme c'est le cas dans la plupart des pays. L'expérience française des cinquante dernières années a montré l'efficacité de ce modèle, qui garantit l'intégration des nouveautés dans un processus de conception validé par l'expérience et qui assure un pilotage des orientations de recherche par les besoins de la pratique. 
Les recherches en géotechnique font intervenir des spécialités très variées : conception de matériel de mesure ou d'essai, méthodes numériques, géophysique, minéralogie, chimie, mécanique, rhéologie... Beaucoup d'entre elles ont des attaches avec des applications dans d'autres domaines. Mais leur centrage sur le champ de la géotechnique est la condition nécessaire de leur succès dans ce domaine. Si l'on ne parle pas le même langage, si l'on n'a pas une perception complète des problèmes à résoudre et des propriétés particulières des matériaux et si l'on n'a pas l'occasion de valider soi-même ses productions sur des problèmes réels, il est difficile d'être connu et reconnu des acteurs d'une discipline qui est aussi un métier. Ou alors, il faut avoir un intermédiaire - interprète, capable de comprendre et restituer les travaux dans le contexte de ce métier.

\section{7}

\section{Géotechnique et enseignement}

Dans le contexte français, la question de la formation des géotechniciens se pose pour l'essentiel audelà du baccalauréat, que ce soit au niveau des BTS/DUT ou des formations de maitrise, d'ingénieurs ou de géologues. Elle peut être discutée sous plusieurs angles : quels géotechniciens veut-on obtenir ? Quel est le contenu des cours et des autres activités nécessaires à l'acquisition d'un métier? Quels enseignants sont à mème de les transmettre ? Quelle formation continue est nécessaire? Quelles sont les meilleures techniques d'enseignement compte tenu de l'origine des étudiants? Les principales composantes de la géotechnique n'existent en tant que discipline que depuis une cinquantaine d'années pour la mécanique des sols. une trentaine pour la mécanique des roches et autant pour la géologie de l'ingénieur. L'organisation des enseignements est une prérogative des établissements, voire des professeurs, mais des commissions techniques des sociétés internationales de mécanique des sols et de géotechnique (SIMSG), de mécanique des roches (SIMR) et de géologie de l'ingénieur et de l'environnement (AIGE) ont été constituées pour essayer d'avoir une meilleure vue d'ensemble du dispositif de formation dans le monde, voire de l'améliorer.

Partir du point de vue des enseignants peut être une clé d'analyse utile, car le système français de gestion des enseignants de l'enseignement supérieur, qui conduit à généraliser des carrières entières dans l'enseignement, sans contacts avec la pratique du métier de l'ingénieur, pose quelques problèmes pour l'enseignement d'un métier comme la géotechnique. Cette difficulté n'existe pas dans les pays, comme l'Allemagne, où les professeurs sont choísis parmi les ingénieurs ayant derrière eux une carrière professionnelle incluant des années de pratique et où il est considéré comme normal qu'ils continuent d'exercer une activité d'études et d'expertises, tout en dirigeant l'enseignement et la recherche.

Les quelques écoles d'ingénieurs qui n'ont pas de corps d'enseignants à titre principal et font systématiquement appel à des professeurs ayant une autre activité professionnelle principale, sont certainement mieux à même d'avoir des enseignants qui connaissent les disciplines et les métiers qu'ils enseignent pour les pratiquer eux-mêmes.
Ceci étant, le contenu de l'enseignement mérite aussi un examen approfondi : la tradition de l'Europe continentale est de former des ingénieurs en génie civil, dont certains acquièrent une spécialisation en géotechnique. Dans certains pays, les géologues n'ont pas le droit (au sens juridique) de signer des projets. En France, la tradition est plus souple et le métier d'ingénieur, et plus encore de géotechnicien, n'est pas défini dans des textes normatifs. Il est donc possible d'exercer le métier de géotechnicien sur la base d'une formation de géologie appliquée. Comme il n'y a pas d'ordre des ingénieurs, ni d'organisation qualifiant les géotechniciens, la responsabilité de reconnaitre la qualification des géotechniciens incombe à leur employeur.

Quand la géotechnique s'insère dans une formation de génie civil, les enquêtes réalisées au cours des dernières années ont conclu que la formation la plus légère comporte un cours de mécanique des sols et un cours de calcul des fondations et soutènements, souvent avec un cours de géologie générale ou appliquée. Cette formation donne aux étudiants une idée des problèmes que peut poser le sol, leur donne des informations sur les types d'ouvrages géotechniques et quelques méthodes de calcul pour les ouvrages courants. Mais elle ne constitue pas une formation au métier de géotechnicien, car elle ne se préoccupe pas du point critique de la géotechnique, qui est la fabrication du modèle géotechnique du site et de l'ouvrage, qui constitue le point de départ des calculs de justification demandés par les normes.

Des formations plus lourdes existent pour ceux qui désirent travailler dans le domaine géotechnique à la fïn de leurs études. On y atteint une centaine d'heures de cours, travaux dirigés et travaux pratiques, voire quelques centaines. Cela permet de donner aux étudiants des informations détaillées dans les différents champs de la géotechnique et de préparer des projets. Ces formations constituent un bon début pour l'apprentissage du métier de géotechnicien, quand elles sont données par des ingénieurs expérimentés. Elles sont aussi une forme de compagnonnage pour ingénieurs, ce qui est l'une des caractéristiques de la formation des artisans.

L'unanimité se fait actuellement sur l'idée que les ingénieurs géotechniciens sortant d'école doivent avoir des bases solides de géologie, mécanique des sols et mécanique des roches et acquérir le sens de la géotechnique, c'est-à-dire les réflexes communs à tous les géotechniciens expérimentés, qui savent quels sont les éléments importants et les pièges possibles dans les données géotechniques et la conception des ouvrages. Les discussions portent sur la quantité de modélisation à introduire dans les programmes et sur les nouvelles techniques, dont les promoteurs aimeraient bien qu'elles deviennent des sujets à part entière, ce qui est difficile dans une ambiance générale de réduction du volume des enseignements techniques.

L'organisation de la formation continue dépend à l'évidence des règles du jeu social : dans les pays comme la France où les diplômes d'ingénieur sont contrôlés, mais pas la qualification professionnelle des personnes qui exercent le métier d'ingénieur, les cours peuvent être organisés de façon assez informelle, sans délivrance de diplômes. Dans les pays où l'enregistrement des ingénieurs est obligatoire, comme les ÉtatsUnis, le Canada ou le Royaume-Uni, des systèmes de validation des cours de formation continue ont été mis 
en place au cours des dernières années. Là encore, la tendance est plutôt au compagnonnage, c'est-à-dire à la transmission de l'expérience d'ingénieurs expérimentés, qu'à des cours académiques.

\section{8}

\section{Conclusion}

A l'issue ce plaidoyer pour l'affirmation et la reconnaissance du caractère intégré de l'exercice de la géotechnique, nous allons simplement rappeler quelques idées qui pourraient servir de base à une politique d'avenir pour ce champ d'activité majeur pour la société :

- la première idée est que la géotechnique est un métier qui s'appuie sur des outils et compétences pluridisciplinaires mais nécessite aussi une expérience acquise sur le terrain, par compagnonnage et par la pratique personnelle d'études et d'expertises. Il nous parait utile que ce métier soit reconnu et que les carrières professionnelles des géotechniciens soient organisées pour leur permettre de progresser continûment en connaissances et en compétences ;

- la deuxième idée est que l'histoire des dernières décennies a montré que le rassemblement dans les mêmes organismes des fonctions d'études, d'expertise, de recherche et d'enseignement crée les conditions du progrès technique, d'une formation efficace des générations suivantes et de la gestion collective du patrimoine des connaissances au service des différentes composantes de la société. Ce type d'organisation, dont l'objectif est l'excellence et une position de référence nationale et mondiale, doit être privilégié et préservé pour assurer le développement permanent de la géotechnique dans ses différents champs d'application ; - la troisième idée est que la place de la géotechnique dans l'élaboration des projets et l'exécution des travaux doit être restaurée, en évitant par-dessus tout le morcellement du suivi géotechnique d'un projet au gré des appels d'offres des phases successives du projet. Le coût des reconnaissances devrait être prévu au niveau adéquat dès les études et les différentes phases de l'étude confiées au mème géotechnicien, afin de permettre l'interaction nécessaire entre les prévisions des études et l'observation de la réalité des sols et des roches pendant les travaux :

- il faut enfin développer les actions de promotion du rôle indispensable de la géotechnique pour les réalisations du génie civil ou plus généralement de l'aménagement des espaces, mais surtout pour la gestion des risques naturels auxquels les sociétés modernes acceptent de moins en moins d'être soumises.

\section{Bibliographie}

Dunnicliff J., Deere D.U. (Ed.) 1984 - Judgement in geotechnical engineering. The professional legacy of R.B. Peck. A Wiley-Interscience Publication. New York. John Wiley and Sons, 332 pages. Norme EN 1997-1 2003 - Calcul géotech- nique. Partie 1: Rèqles générales. Comité européen de normalisation (CEN) et Associations nationales de normalisation (A paraitre).

Fascicule 62 - Titre V. 1993. Règles techniques de conception et de calcul des fon. dations des ouvrages de génie civil. Cahier des clauses techniques générales applicables aux marchés publics de travaux. Fascicule No62. Titre V Ministère de I'Équipement, du Logement et des Transports, textes officiels, $n^{\circ} 93 / 3,182$ pages, 